\title{
$\mathrm{Cr}$ 在 $\mathrm{Fe}-\mathrm{C}$ 奥氏体中的价电子结构及 其在相变动力学中的行为*
}

\author{
戴天时 刘志林张瑞林 \\ （吉林工业大学应用物理系,长春）（吉林大学物理系,长春）
}

关词 Cr、奥氏体、相变行为

\section{一、 $\mathrm{Fe}-\mathrm{C} 、 \mathrm{Fe}-\mathrm{C}-\mathrm{Cr}$ 㡀氏体的价电子结构}

按文献 $[1,2]$, 本文建立了含 $0.15-2 \% \mathrm{C}$ 的 $\mathrm{Fe}-\mathrm{C}$ 奥氏体的价电子结构, 见表 1。依文 献 [3], $\mathrm{Cr}$ 在 $\mathrm{Fe}-\mathrm{C}$ 奥氏体中应呈 $\mathrm{C}-\mathrm{Cr}$ 偏聚状态, 根据文献 $[3,4]$ 的方法, 本文又建立了 $\mathrm{Fe}-\mathrm{C}-\mathrm{Cr}$ 奥氏体的价电子结构. 为了说明符号 $n^{l j}$ 的意义, 文中给出了含 $0.3 \% \mathrm{C}-\mathrm{Cr}$ 晶胞的 价电子结构 (见表 2), 其余 $\mathrm{Fe}-\mathrm{C}-\mathrm{Cr}$ 奥氏体的价电子结构见表 1。作者认为表 2 中 C 与 $\mathrm{Fe}^{i}$ 、 $\mathrm{Cr} 、 \mathrm{Fe}^{e}$ 共价键上的共用电子对构成了对 C 原子扩散的阻力, 其大小可用 $\boldsymbol{n}_{c}^{D}$ 来表示 $\left(\boldsymbol{n}_{::}^{D}-\right.$ $\left.n_{A}+n_{B}+n_{G}\right)$. 由于本文的讨论中只涉及最强键 $D_{(s, d)}^{C-x}$ 上的 $n_{A}$ 值, 因此表 1 中仅汇集了原 子状态 $\sigma, n_{A}$ 和 $n_{c}^{D}$ 的值和讨论中用到的 $n_{B}$ 值.

\section{二、 $\mathrm{Cr}$ 在相变动力学中的行为}

比较表 1 中的 $n_{A}$ 值发现:

1. 含 C 量小于 $0.6 \%$ 时, $\mathrm{Fe}-\mathrm{C}-\mathrm{Cr}$ 结构单元 $D_{i=1}^{C \mathrm{Pd} d}$ 键上的 $n_{A}$ 值比 $\mathrm{Fe}-\mathrm{C}$ 结构单元 $D_{(-1)}^{C}$ Pd 键上的 $n_{A}$ 值小, 按文献[5], Fe-C-Cr 合金的 C-曲线应在中温转变区的上部形成河 湾. 这一微观固态信息和实际相符.

2. 含 C 量大于 $0.6 \%$ 㖇小于 $1.4 \%$ 时, $\mathrm{Fe}-\mathrm{C}-\mathrm{Cr}$ 结构单元 $D_{\mathrm{in}}^{\mathrm{C}}-\mathrm{C}_{\mathrm{r}}$ 键上的 $n_{1}$ 值大于 $\mathrm{Fe}-\mathrm{C}$

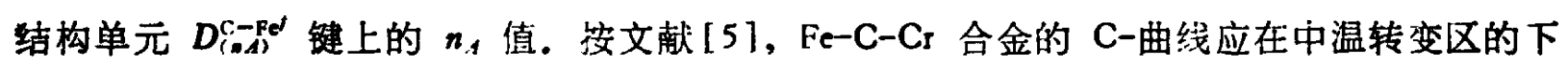
部形成河湾. 这一价电子结构的微观固态反应也与实际相符.

3. 含 C 量在 $1.5-2.0 \%$ 之间时, 尽管 $\mathrm{Fe}-\mathrm{C}-\mathrm{Cr}$ 结构单元 $D_{\mathrm{inA}}-\mathrm{Cr}$ 键上的 $n_{A}$ 值较 $\mathrm{Fe}-\mathrm{C}$ 结 构单元 $D_{(n A)}^{C-F d}$ 键上的 $n_{A}$ 值大, 但 Fe-C-Cr 结构单元 $D_{(n B)}^{(-r) d}$ 键上的 $n_{B}$ 值却小得多, 其等效 键上的共用电子对 $\bar{n}-\frac{1}{3}\left[n_{A}+2 n_{B}\right]$ 比 $\mathrm{Fe}-\mathrm{C}$ 结构单元的 $\bar{n}-\frac{1}{3}\left[3 n_{A}\right]$ 为小。依文献 $[5]$ 又应在中温转变区的上部形成河湾. 当 $\mathrm{Cr}$ 的含量很高时, 例如 $\mathrm{Cr} 12$, 由于 $\mathrm{Fe}-\mathrm{C}-\mathrm{Cr}$ 结构 单元的权重增加, 河湾可消失而使 C-曲线只有一个算子。这一微观固态信息也与实际相符。

本文 1988 年8 月15 日收到. 1989 年3月27 日收到堡改程.

- 国家自然科学慕金资助项目. 
表 $1 \mathrm{Fe}-\mathrm{C} 、 \mathrm{Fe}-\mathrm{C}-\mathrm{Cr}$ 晶胞的 $\sigma \cdot n_{A}$ 及 $\boldsymbol{*}_{\mathrm{C}}^{\mathrm{D}}$ 值

\begin{tabular}{|c|c|c|c|c|c|c|}
\hline \multirow{2}{*}{$\frac{C}{(w t)}$} & \multicolumn{3}{|c|}{$\mathrm{Fe}-\mathrm{C}$} & \multicolumn{3}{|c|}{$\mathrm{Fe}-\mathrm{C}-\mathrm{Cr}$} \\
\hline & $\boldsymbol{\sigma}$ & $n_{A}$ & $n q$ & $\sigma$ & $n_{A}$ & $n q$ \\
\hline 0.15 & $\mathrm{Fe}_{1, s}^{c}, \mathrm{Fe}_{1}^{t}, \mathrm{C}_{6}$ & 1.1035 & 1.1192 & $\mathrm{Fe}_{1,}^{c}, \mathrm{Fe}_{1,}^{\prime}, \mathrm{C}_{0}, \mathrm{Cr}_{\mathrm{r}_{1}}$ & 1.0478 & 2.0565 \\
\hline 0.20 & $\mathrm{Fe}_{14}^{c}, \mathrm{Fe}_{16}^{t}, \mathrm{C}_{6}$ & 1.0671 & 1.0823 & $\mathrm{Fe}_{1}^{i}, \mathrm{Fe}_{10}^{1}, \mathrm{C}_{6}, \mathrm{Cr}_{3}$ & 1.0463 & 2.0729 \\
\hline 0.30 & $F e_{1 s}^{c}, F_{1 s}^{\prime}, C_{6}$ & 1.0158 & 1.0289 & $\mathrm{Fe}_{i s}^{c}, \mathrm{Fe}_{i}^{\prime}, \mathrm{C}_{\theta}, \mathrm{Cr}_{\mathrm{s}}$ & 1.0101 & 1.9990 \\
\hline 0.40 & $F_{i s}^{c}, F_{t s}^{\prime}, C_{6}$ & 1.0075 & 1.0205 & $\mathrm{Fe}_{1,}^{c}, \mathrm{Fe}_{1,}^{\prime}, \mathrm{C}_{6}, \mathrm{Cr}_{3}$ & 0.9778 & 1.8989 \\
\hline 0.45 & $F e_{13}^{c}, F_{1}^{f}, C_{6}$ & 1.0101 & 1.0230 & $\mathrm{Fe}_{13}^{c}, \mathrm{Fe}_{1,}^{\prime}, \mathrm{C}_{6}, \mathrm{Cr}_{r}$, & 0.9618 & $1.90+0$ \\
\hline 0.50 & $\mathrm{Fe}_{13}^{c}, \mathrm{Fe}_{1,}^{\prime}, \mathrm{C}_{6}$ & 1.0122 & 1.0250 & $\mathrm{Fe}_{2,}^{c}, \mathrm{Fe}_{1,}^{t}, \mathrm{C}_{0,}, \mathrm{Cr}_{\mathrm{r}}$ & 0.9638 & 1.9078 \\
\hline 0.60 & $\mathrm{Fe}_{13}^{\mathrm{f}}, \mathrm{Fe}_{1, t}^{t}, \mathrm{C}_{6}$ & 1.0153 & 1.0280 & $\mathrm{Fe}_{14}^{c}, \mathrm{Fe}_{16}^{\prime}, \mathrm{C}_{6}, \mathrm{Cr}_{6}$ & 1.0029 & 1.9469 \\
\hline 0.70 & $\mathrm{Fe}_{i s}, \mathrm{Fe}_{14}^{\prime}, \mathrm{C}_{\text {. }}$ & 0.9236 & 0.9345 & $\mathrm{Fe}_{16}^{c}, \mathrm{Fe}_{17}^{\prime}, \mathrm{C}_{0,}, \mathrm{Cr}_{\mathrm{r}_{12}}$ & 1.3305 & 2.2331 \\
\hline 0.80 & $\mathrm{Fe}_{1,}^{c}, \mathrm{Fe}_{14}^{\prime}, \mathrm{C}_{6}$ & 0.9250 & 0.9358 & $\mathrm{Fe}_{16}^{c}, \mathrm{Fe}_{17}^{\prime}, \mathrm{C}_{6,}, \mathrm{Cr}_{13}$ & 1.3428 & 2.2370 \\
\hline 0.90 & $\mathrm{Fe}_{13}^{\mathrm{c}}, \mathrm{Fe}_{14}^{\prime}, \mathrm{C}_{6}$ & 0.9262 & 0.9370 & $\mathrm{Fe}_{16}^{c}, \mathrm{Fe}_{1,}^{\prime}, \mathrm{C}_{6}, \mathrm{Cr}_{r_{13}}$ & 1.3444 & 2.2397 \\
\hline 1.00 & $F e_{13}^{f}, F_{14}^{\prime}, C_{6}$ & 0.9271 & 0.9379 & $\mathrm{Fe}_{i 4}^{c}, \mathrm{Fe}_{i,}^{\prime}, \mathrm{C}_{6}, \mathrm{Cr}_{8}$ & 1.0257 & 1.9587 \\
\hline 1.10 & $\mathrm{Fe}_{13}^{c}, \mathrm{Fe}_{14}^{\prime}, \mathrm{C}_{0}$ & 0.9278 & 0.9385 & $\mathrm{Fe}_{13}^{c}, \mathrm{Fe}_{1,1}^{\prime}, \mathrm{C}_{6}, \mathrm{Cr}_{1}$ & 1.0015 & 1.9278 \\
\hline 1.20 & $\mathrm{Fe}_{13}^{\mathrm{f}}, \mathrm{Fe}_{1,}^{\prime}, \mathrm{C}_{0}$ & 0.9284 & 0.9391 & $\mathrm{Fe}_{13}^{c}, \mathrm{Fe}_{1 s}^{\prime}, \mathrm{C}_{0}, \mathrm{Cr}_{r}$ & 1.0021 & 1.9290 \\
\hline 1.30 & $\mathrm{Fe}_{13}^{c}, \mathrm{Fe}_{14}^{\prime}, \mathrm{C}_{6}$ & 0.9289 & 0.9396 & $\mathrm{Fe}_{13}^{c}, \mathrm{Fe}_{1,}^{\prime}, \mathrm{C}_{6}, \mathrm{Cr}_{\mathrm{r}}$ & 1.0026 & 1.9221 \\
\hline 1.40 & $\mathrm{Fe}_{\mathrm{i} 3}^{\mathrm{c}}, \mathrm{Fe}_{i 4}^{i}, \mathrm{C}_{6}$ & 0.9293 & 0.9400 & $\mathrm{Fe}_{i 3}^{c}, \mathrm{Fe}_{i s}^{\prime}, \mathrm{C}_{4}, \mathrm{Cr}_{r_{3}}$ & 1.0111 & 1.9310 \\
\hline 1.50 & $\mathrm{Fec}_{13}^{c}, \mathrm{Fe}_{14}^{\prime}, \mathrm{C}_{8}$ & 0.9296 & 0.9403 & $\mathrm{Fe}_{1,}^{\mathrm{f}}, \mathrm{Fe}_{14}^{\prime}, \mathrm{C}_{0}, \mathrm{Cr}_{r_{1}}$ & $\mid \begin{array}{l}0.9575 \\
0.8187\left(n_{B}\right)\end{array}$ & 1.7873 \\
\hline 1.60 & $F e_{\text {is }}^{c}, F_{14}^{\prime}, C_{0}$ & 0.9299 & 0.9406 & $\mathrm{Fe}_{13}^{c}, \mathrm{Fe}_{14}^{\prime}, \mathrm{C}_{6}, \mathrm{Cr}_{1}$ & $\left|\begin{array}{ll}0.9578 & \\
0.8189 & \left(*_{8}\right)\end{array}\right|$ & 1.7877 \\
\hline 1.70 & $\mathrm{Fe}_{13}, \mathrm{Fe}_{14}^{\prime}, \mathrm{C}_{6}$ & 0.9301 & 0.9408 & $\mathrm{Fe}_{13}^{c}, \mathrm{Fe}_{14}, \mathrm{C}_{6}, \mathrm{Cr}_{\mathrm{r}_{1}}$ & $\left|\begin{array}{ll}0.9581 & \\
0.8191 & \left(n_{B}\right)\end{array}\right|$ & 1.7882 \\
\hline 1.80 & $\mathrm{Fe}_{i 3}^{i_{3}}, \mathrm{Fe}_{14}^{\prime}, \mathrm{C}_{0}$ & 0.9303 & $0.940 i$ & $\mathrm{Fe}_{13}^{c_{13}}, \mathrm{Fe}_{1}^{\prime}, \mathrm{C}_{0}, \mathrm{C}_{r_{1}}$ & $\left|\begin{array}{ll}0.9583 & \\
0.8193 & \left(n_{8}\right)\end{array}\right|$ & 1.7886 \\
\hline 1.90 & $\mathrm{Fe}_{\mathfrak{1},}^{c}, \mathrm{Fe}_{\mathfrak{s},}^{\prime}, \mathrm{C}_{\bullet}$ & 0.9305 & $0.9+12$ & $\mathrm{Fe}_{13}, \mathrm{Fe}_{14}^{\prime}, \mathrm{C}_{0}, \mathrm{Cr}_{\mathrm{r}_{1}}$ & $\left|\begin{array}{l}0.9584 \\
0.8195\left(n_{B}\right)\end{array}\right|$ & 1.7889 \\
\hline 2.00 & $\mathrm{Fe}_{13}^{c}, \mathrm{Fe}_{142}^{\prime}, \mathrm{C}_{6}$ & 0.9307 & 0.9414 & $\mathrm{Fe}_{13}, \mathrm{Fe}_{14}, \mathrm{C}_{4}, \mathrm{Cr}_{r_{1}}$ & $\left|\begin{array}{ll}0.9586 & \\
0.8196 & \left(m_{B}\right)\end{array}\right|$ & 1.7892 \\
\hline
\end{tabular}

4. $\mathrm{Cr}$ 的加人使 $\mathrm{Fe}-\mathrm{C}-\mathrm{Cr}$ 结构单元的 $n_{c}^{D}$ 值比 $\mathrm{Fe}-\mathrm{C}$ 结构单元的 $\boldsymbol{n}_{c}^{D}$ 值大得多, 这是 $\mathrm{Cr}$ 使 C一曲线右移，特别是使珠光体的畕子右移的本质原因.

5. 对于含 $\mathrm{C}$ 量小于 $0.6 \%$ 的 $\mathrm{Fe}-\mathrm{C}$ 合金,加人 $\mathrm{Cr}$ 可提高淬透性，而对含 $\mathrm{C}$ 量在 $0.6-1.4 \%$ 之间的 Fe-C 合金, $\mathrm{Cr}$ 的加人对淬透性就不十分有效,因为它的 C-曲线河湾在下部; 而对含 C 量大于 $1.5 \%$ 的合金, $\mathrm{Cr}$ 的加人又提高了淬透性。这一价电子结构与化学成份的关系使得 Cr 对淬透性的影响十分明了。

\section{三、讨 论}

过去, 人们只是从原子排布及其变化规律的框架研究相变, 对凝聚态原子价电子结构对固 态相变的影响研究得很少. 最近作者对常用合金元素与 Fe、C 凝聚态原子的价电子结构进 行了计算, 发现凝聚态原子的价电子结构对固态相变的影响十分引人入胜. 如能像本文那样 建立各种元素在钢中的价电子结构,那么就可从理论上进行合金成分的设计. 
表 2 含 C-Cr 晶胞 BLD 分析

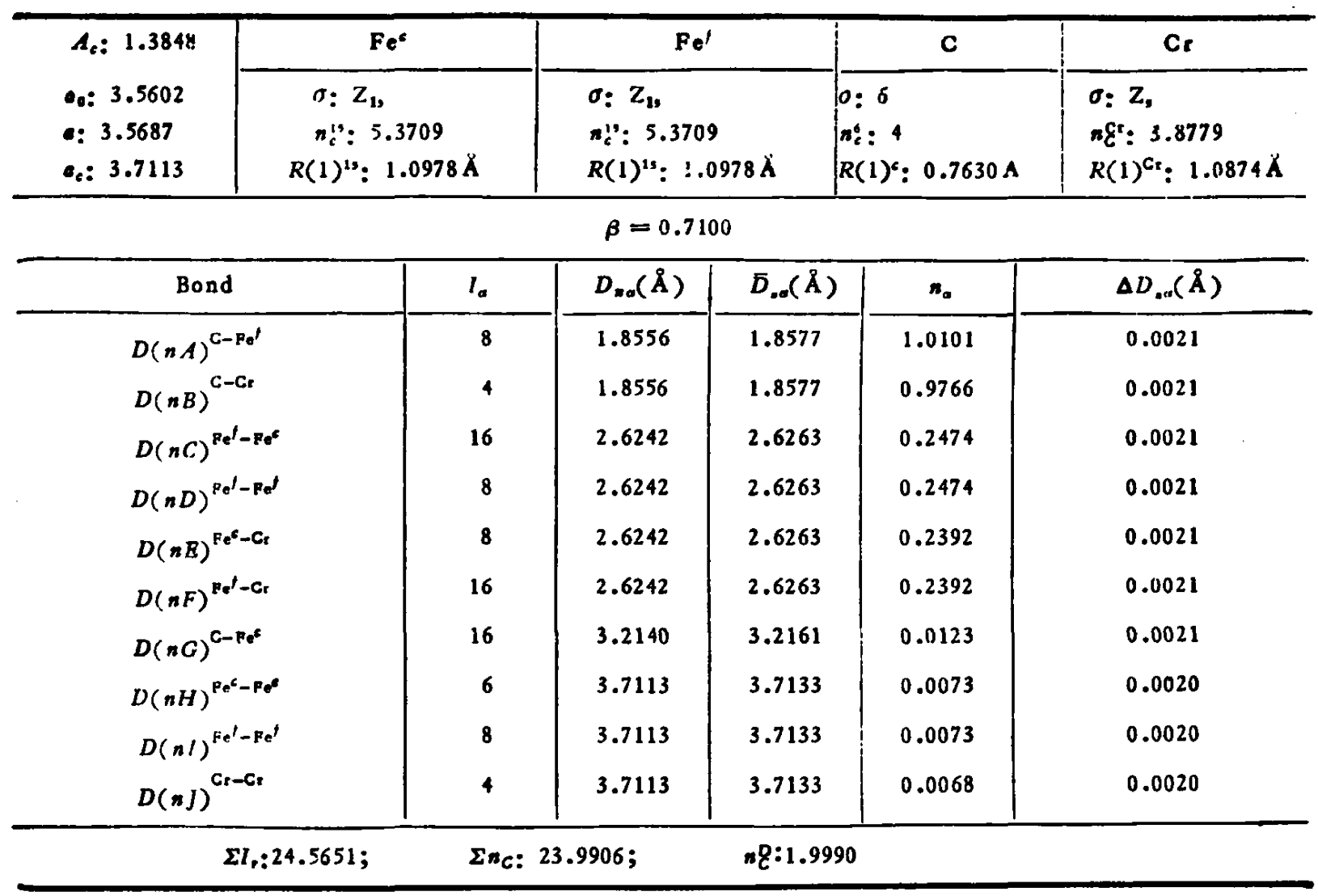

致谢: 本文在余瑞璜先生的指导下完成，作者谨致深切的谢意.

\section{考文城}

[1] 余瑞珙、张瑞林,吉林大学自然科学学报, 1984,51.

[2] 余理璜,科学通报,23(1978),4: 271.

[3] 刘志林,科学通报,32(1987),23: 1787.

[4] 余瑞璜、刘志林,金属科学与工艺,7(1988), 1.

[5]邚志林、筬天时,科学通报,34(1989), 1: 15 。 